\title{
Extended Split-Step Fourier Transform Approach for Accurate Characterization of Soliton Propagation in a Lossy Optical Fiber
}

\author{
Neveen G. A. Farag $\mathbb{D}^{1}{ }^{1}$ Ahmed H. Eltanboly, ${ }^{1}$ M. S. El-Azab, ${ }^{1}$ and S. S. A. Obayya ${ }^{2,3}$ \\ ${ }^{1}$ Mathematics and Engineering Physics Department, Faculty of Engineering, Mansoura University, Mansoura 35516, Egypt \\ ${ }^{2}$ Centre for Photonics and Smart Materials, Zewail City of Science and Technology, October Gardens, 6th of October City, \\ Giza 12578, Egypt \\ ${ }^{3}$ Electronics and Communications Engineering Department, Faculty of Engineering, Mansoura University, Mansoura 35516, Egypt
}

Correspondence should be addressed to Neveen G. A. Farag; eng_neveen@hotmail.com

Received 1 December 2021; Revised 31 January 2022; Accepted 17 February 2022; Published 8 March 2022

Academic Editor: Youssri Hassan Youssri

Copyright (c) 2022 Neveen G. A. Farag et al. This is an open access article distributed under the Creative Commons Attribution License, which permits unrestricted use, distribution, and reproduction in any medium, provided the original work is properly cited.

\begin{abstract}
In this paper, we present a novel extension of the well-known split-step Fourier transform (SSFT) approach for solving the onedimensional nonlinear Schrödinger equation (NLSE), which incorporates the fiber loss term. While this essential equation governs the pulse propagation in a lossy optical fiber, it is not supported by an exact analytical solution. In this regard, extended versions of the Fourier pseudospectral method (FPSM) and Hopscotch method (HSM) are effectively established as well to cope with the fiber losses effects associated with the pulses' propagation through the fiber optics, and thus, numerous comparisons are exhaustively conducted among these three compelling numerical approaches to validate their reliability, stability, and accuracy. Based on this, the MATLAB numerical findings bolster that the extended version of the SSFT approach demonstrates superior performance over the other suggested schemes in simulating the solitons propagation in a lossy optical fiber.
\end{abstract}

\section{Introduction}

A soliton, or what is also known as a solitary wave, is a selfreinforcing wave packet that sustains both its form and velocity while propagation, regardless of the travelling distance or presence of obstacles. This shape conservation property makes it potentially compatible with the longdistance expansive data transmission [1]. Moreover, a cancellation of nonlinear and dispersive effects in the propagation medium, which is a basic feature that invariably occurs in the optical fibers, causes solitons. In other words, the term soliton refers to any optical pulse that resists changing when it is transferred from the source to the destination due to a sensitive balance between the nonlinear and linear effects in the medium [2]. Therefore, the solitons are utterly beneficial in transporting information through optical fibers in a variety of modern communication systems because they demonstrate a robust grasp in achieving high bit rate transmission while minimizing error possibilities. This occurs due to their propagation without any distortion or shape chang- ing while moving through a lossless medium, which guarantees retaining the information stored in them until they reach the desired location [3]. This means that solitons pulses have incredibly stable characteristics in propagation through the transmission path due to its powerful resistance to the distortion effects, which resulted from the nonlinearity and dispersion and inherited in the optical fibers. However, the only serious damage that may change their shapes while propagation is the attenuation caused by the fiber losses. Thus, numerous researchers have suggested to compensate these fiber losses by the aid of the amplification in order to mitigate the functionality of the all-optical transmission system [4].

The optical fiber, where the solitons always propagate, is an extremely fine and thin pure glass that is usually made of pure silica. This fascinating fiber simulates the function of a waveguide in transmitting light pulses through the fiber ends because it comprises a transparent core surrounded by a lower refraction index transparent cladding material; this unique structure, presented in Figure 1 [5], confines the light 


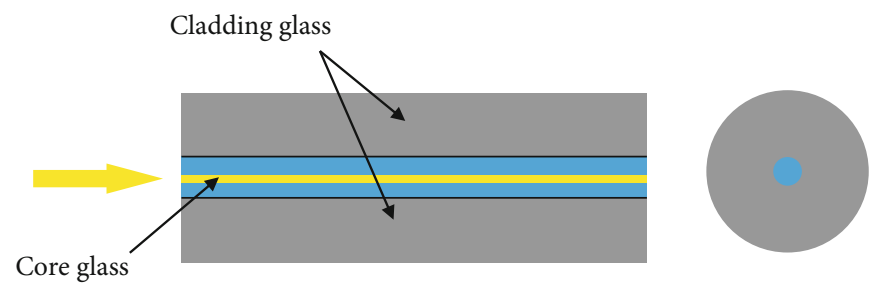

Figure 1: A schematic diagram of a single-mode fiber optics.

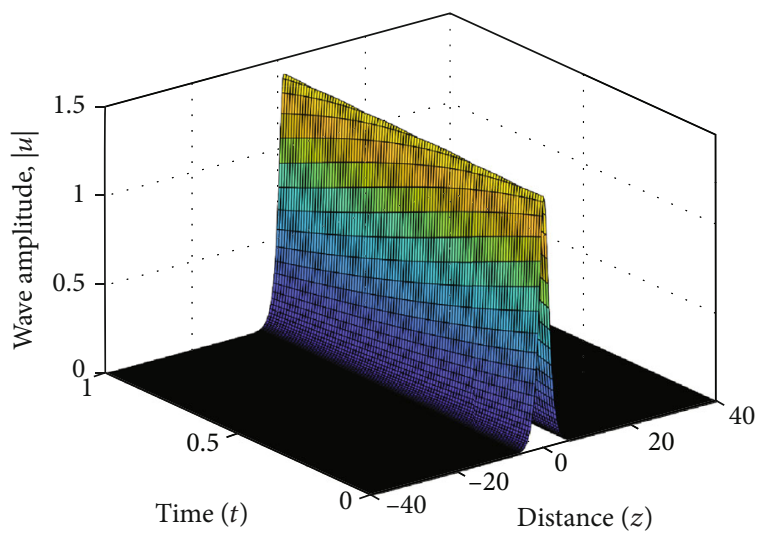

FIgure 2: A 3D plot of the 1D CNLSE exact solution.

wave in the core region in accordance with the total internal reflection phenomenon. Hence, optical fibers are eminently used in the communications field, especially for long distance and higher band width transmission. In contrast, transmission through the metal wires experiences higher losses and encounters electromagnetic interference, which negatively impacts the quality of the transmitted data [6].

The propagation of the optical pulses through the optical fiber, which is a nonlinear medium, is modeled by the 1D nonlinear Schrödinger equation (NLSE). Thus, this crucial second-order partial differential equation is highly compatible with depicting the unidirectional propagation of the light waves in single-mode fiber optics [7]. More importantly, although it is remarkably noticed that the NLSE can be solved using both exact and numerical solutions, its analytical solution is extremely tough and tedious to be found, abreast of being provided for a limited set of initial conditions [8]. As a result of this, the numerical solutions of the NLSE frequently demonstrate a significant role in solving and approximating the equation and, likewise, understanding the equation's physical behavior [9].

Moreover, the exact analytical solutions of NLSE can only be obtained in the case of the soliton solutions, if and only if the attenuation term is neglected, whereas, for the other solutions, which are not solitons, or in the presence of fiber losses that must occur in any realistic optical fiber, the exact solutions are not easily to be reached. In other words, the exact analytical solution can only be found for some specific input pulses, most commonly, the hyperbolic secant and tangent functions. Therefore, the numerical solutions are widely used to unravel this equation. More specifically, the split-step Fourier transform is suggested for its high processing speed, stability, and accuracy, along with other techniques such as the Fourier pseudospectral method and the Hopscotch approach, as employed elsewhere for solving other equations [10-16], which will be comprehensively explained within this framework. Besides, other numerical approaches have recently been developed to report the 1D NLSE by several authors, for more details, see [17-26]. Additionally, analytical approximations to solve the NLSE might exist by implementing plenty of linearization techniques. For instance, perturbation methods tailored for modulation instability, small-signal analysis, variational method, and an approach based on Volterra series, all these methods can provide accurate approximations for any arbitrary modulated input signal $[17,18]$.

On the other hand, opting for a numerical approach to solve this equation should be classified under two main categories, which are either the finite difference methods (FDMs) or the function approximation methods (FAMs), which are divided into both the spectral and pseudospectral methods. In the FDMs, the unknown function is approximated at discrete points in the space-time plane, while replacing the partial derivatives with difference relations employed by Taylor series. Whereas in the FAMs, the basic goal is to approximate the exact solution using an appropriate chosen basis function, a trigonometric function is usually selected. This notable strategy eventually constitutes the finite element method that is categorized as a spectral method [27]. Furthermore, the main distinguished difference between the pseudospectral and spectral methods is that the pseudospectral methods are computed in a discrete space, which resembles the behavior of the FDMs [28, 29].

In this paper, the aim is to seek feasible solutions for the NLSE, incorporating the fiber loss term. More specifically, due to the harmful impact of the fiber losses on changing the authentic form of the transmitted pulse that can obviously be noticed as an attenuation in both the pulse's amplitude and power, an urgent need has emerged to include the fiber losses in our consideration. Furthermore, this attenuation should efficiently be measured and hence compensated using amplifiers in order not to hinder the accurate reception of the pulses at the destination. In other words, including the loss term to our attention in the simulation of the soliton propagation is extremely vital, as recently followed elsewhere [30]. Otherwise, numerical errors may arise, which shall likely lead to biased results that do not genuinely represent the physical phenomenon. For example, failing to correctly model the degradation of the pulse's amplitude and power due to the attenuation was caused by the loss effects. Despite the losses problem in 

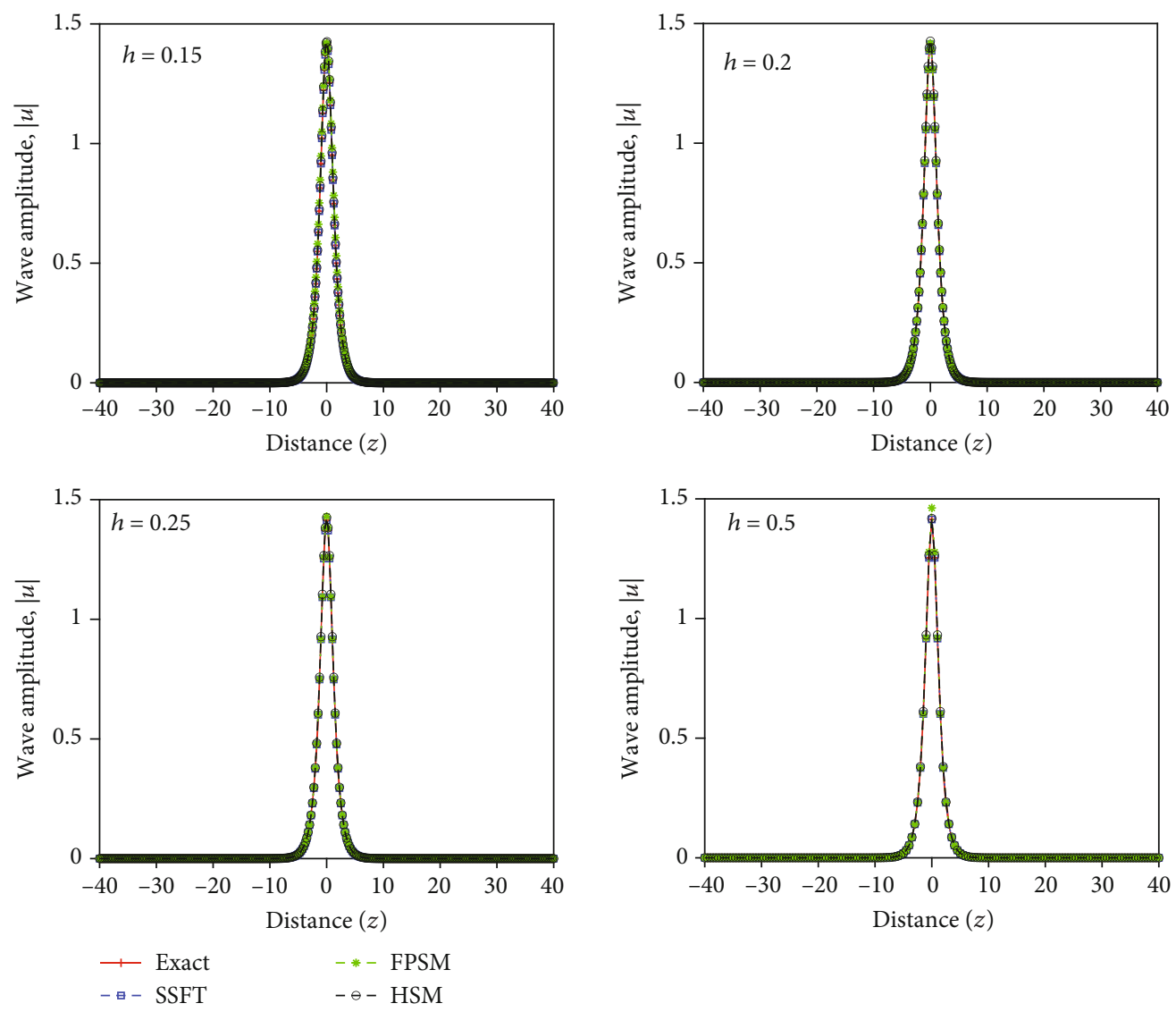

FIGURE 3: A comparison of the split-step Fourier transform (SSFT), the Fourier pseudospectral method (FPSM), the Hopscotch method (HSM), and the exact solution at $\tau=0.001$ and $t=20$ over a space domain $z$ from -40 to 40 , whereas using different values of $h$.

optical fibers has previously attracted a great deal of interest, we exhibit in the present work, for the first time, a method that renders the least sum of square errors along with the fastest computational speed based on an extended version of a well-known split-step strategy. Therefore, our primary target was the meticulous inclusion of the loss term in our suggested numerical methods named split-step Fourier transform (SSFT), Fourier pseudospectral method (FPSM), and Hopscotch method (HSM); the first two methods are pseudospectral methods, while the last one is an explicit finite difference method. Based on this, an extension for each of the three proposed approaches was developed so that their approximate solutions agree with the effects generated by the fiber losses, thereby broadening the validity range of the suggested schemes. Furthermore, in an attempt to substantiate the performance of the extended approaches, plenty of numerical assessments were conducted to track the behavior of both the pulse's shape and power. In this regard, we fundamentally focus on the bright and dark one soliton propagation in fiber optics abreast of demonstrating and testing each of the suggested methods of solutions.

This paper is organized as follows. Following this introduction, which is illustrated in Section 1, the mathematical preliminaries are explained in detail in Section 2. Section 3 is devoted to annotating the modified numerical approaches, while Section 4 elaborates on the error and convergence dis- cussion. In Section 5, the numerical results that were achieved are exhibited, rigorously highlighting the attenuation effect caused by the presence of the fiber losses through the drawing of copious graphs and illustrative comparisons. Eventually, Section 6 culminates with the overall conclusion that summarizes the research work presented in this paper.

\section{Mathematical Preliminaries}

2.1. The NLSE for Lossy Optical Fiber. The 1D NLSE, which governs the pulse propagation in the $z$ direction through a lossy optical fiber, comprises four rudimentary terms, the first-order spatial partial derivative term, the second-order temporal partial derivative term, the nonlinear term, and the unknown function term. Moreover, this outstanding equation mainly relies on some crucial parameters such as the Kerr nonlinear coefficient, the power attenuation constant, and the group velocity dispersion parameter, as illustrated by either Equation (1) or (2):

$$
\frac{\partial u(z, t)}{\partial z}=-i \frac{\beta_{2}}{2} \frac{\partial^{2} u(z, t)}{\partial t^{2}}+i \gamma|u(z, t)|^{2} u(z, t)-\frac{\alpha}{2} u(z, t)
$$



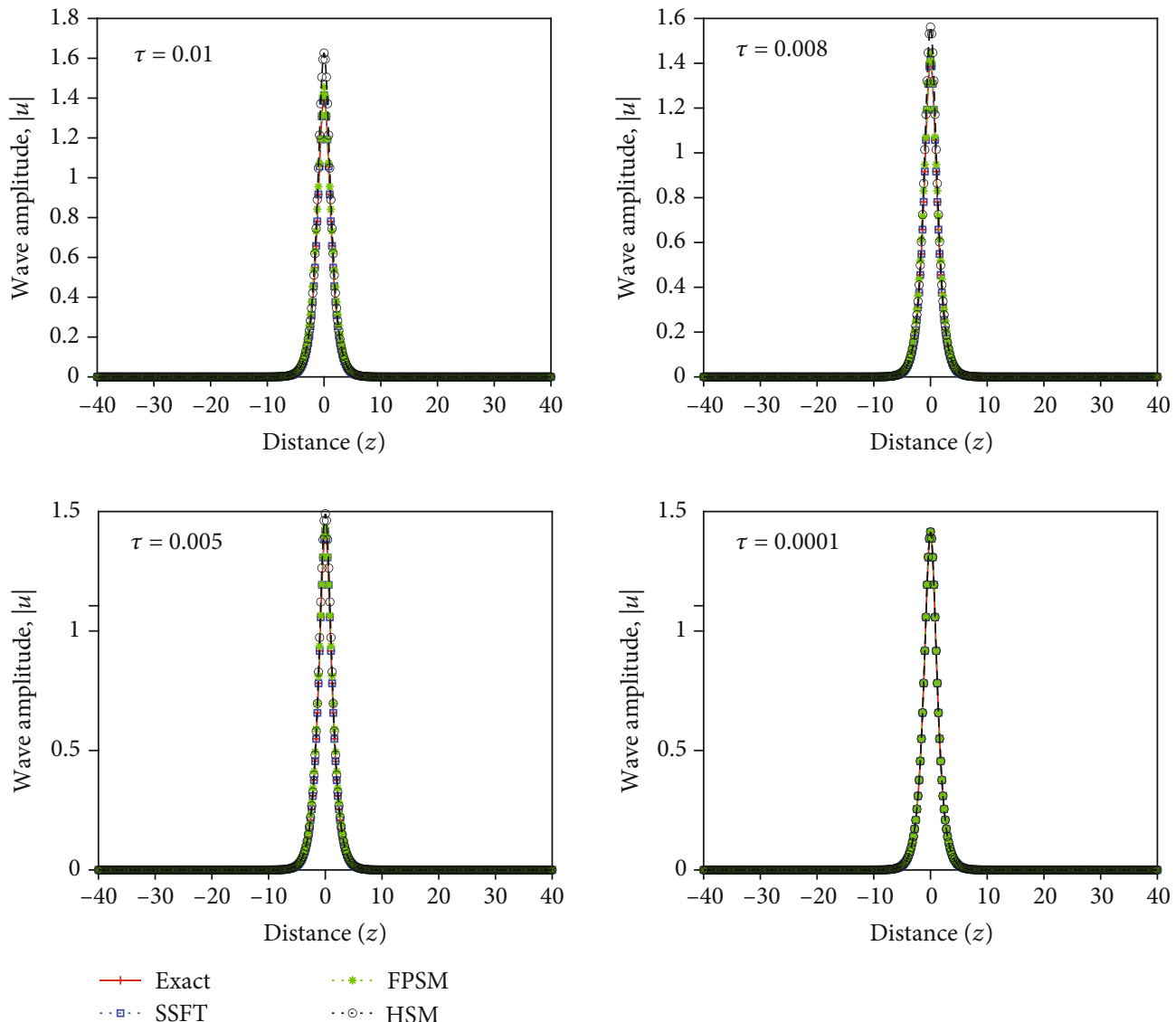

FIGURE 4: A comparison of the split-step Fourier transform (SSFT) and the Fourier pseudospectral method (FPSM), the Hopscotch method (HSM), and the exact solution at $h=0.2$ and $t=20$ over a space domain $z$ from -40 to 40 , whereas using different values $\tau$.

TABLE 1: A comparison among the sum of squares error (SSE) of the split-step Fourier transform (SSFT), the Fourier pseudospectral method (FPSM), and the Hopscotch scheme (HSM), computed for different time values $t$ from 35 to 105. When $h=0.2$ and $\tau=0.001$.

\begin{tabular}{|c|c|c|c|c|c|c|}
\hline Time, $t$ & SSE-SSFT & CPU time (s) & SSE-FPSM & CPU time (s) & SSE-HSM & CPU time (s) \\
\hline 35 & $1.6336 e-21$ & 2.7994 & $2.7847 e-03$ & 2.8612 & $6.5206 e-03$ & 3.7639 \\
\hline 45 & $2.6725 e-21$ & 3.2639 & $4.6158 e-03$ & 3.6818 & $1.1120 e-02$ & 4.8691 \\
\hline 55 & $3.914 e-21$ & 3.9879 & $6.9139 e-03$ & 4.3797 & $1.7156 e-02$ & 5.9605 \\
\hline 65 & $5.439 e-21$ & 4.8138 & $9.6827 e-03$ & 5.2361 & $2.4774 e-02$ & 6.9654 \\
\hline 75 & $7.2441 e-21$ & 5.4023 & $1.2926 e-02$ & 5.884 & $3.4139 e-02$ & 8.0312 \\
\hline 85 & $9.2143 e-21$ & 6.1936 & $1.6647 e-02$ & 6.6282 & $4.5442 e-02$ & 9.2682 \\
\hline 95 & $1.141 e-20$ & 6.9974 & $2.0851 e-02$ & 7.4995 & $5.8899 e-02$ & 10.181 \\
\hline 105 & $1.3794 e-20$ & 7.8761 & $2.5540 e-02$ & 8.5734 & $7.4759 e-02$ & 11.307 \\
\hline
\end{tabular}

TABLE 2: A comparison among the sum of squares error (SSE) of the split-step Fourier transform (SSFT), the Fourier pseudospectral method (FPSM), and the Hopscotch scheme (HSM), computed for different time values $t$ from 35 to 105. When $h=0.2$ and $\tau=0.0001$.

\begin{tabular}{lcccccc}
\hline Time, $t$ & SSE-SSFT & CPU time $(\mathrm{s})$ & SSE-FPSM & CPU time $(\mathrm{s})$ & SSE-HSM & CPU time $(\mathrm{s})$ \\
\hline 35 & $1.4036 e-19$ & 24.941 & $2.7610 e-05$ & 27.572 & $5.9491 e-05$ & 36.65 \\
45 & $2.3119 e-19$ & 32.435 & $4.5653 e-05$ & 36.867 & $9.8615 e-05$ & 46.599 \\
\hline
\end{tabular}


TABLE 3: A comparison among the sum of squares error (SSE) of the split-step Fourier transform (SSFT), the Fourier pseudospectral method (FPSM), and the Hopscotch scheme (HSM), computed for different time values $t$ from 35 to 105 . When $h=0.2$ and $\tau=0.008$.

\begin{tabular}{lcccccc}
\hline Time, $t$ & SSE-SSFT & CPU time $(\mathrm{s})$ & SSE-FPSM & CPU time $(\mathrm{s})$ & SSE-HSM & CPU time $(\mathrm{s})$ \\
\hline 35 & $1.9435 e-23$ & 0.35126 & $1.9046 e-01$ & 0.43022 & $1.5158 e+00$ & 0.63134 \\
45 & $3.1307 e-23$ & 0.44921 & $3.2239 e-01$ & 0.51259 & $1.0684 e+01$ & 0.70445 \\
\hline
\end{tabular}

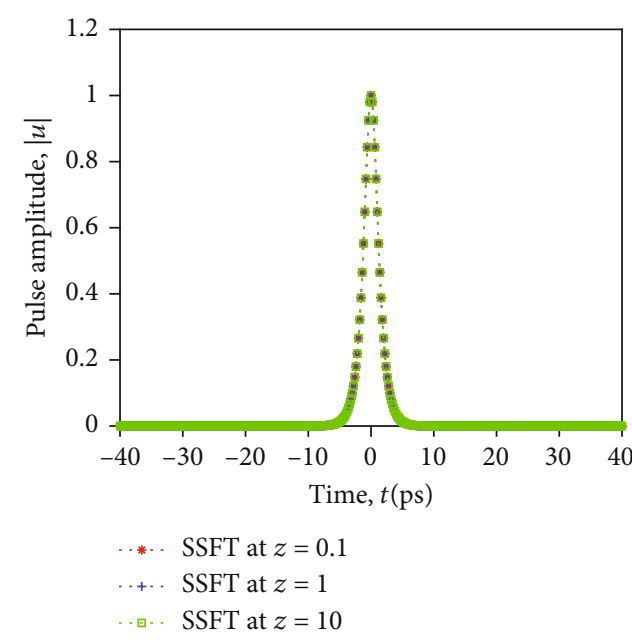

(a)

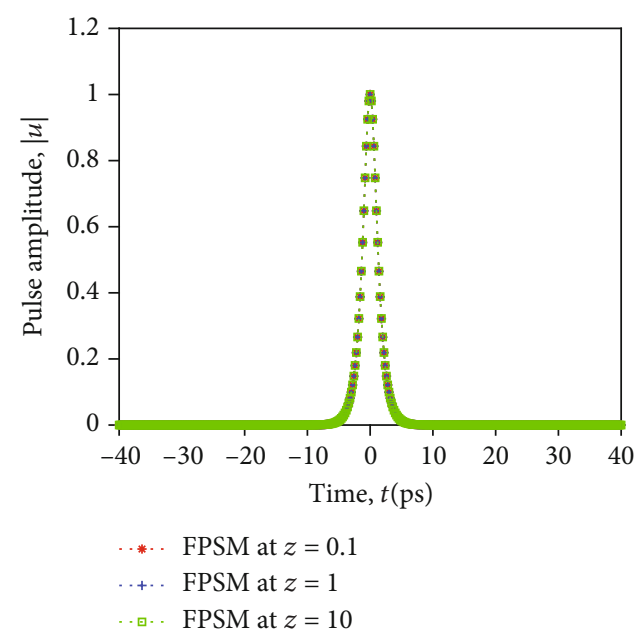

(b)

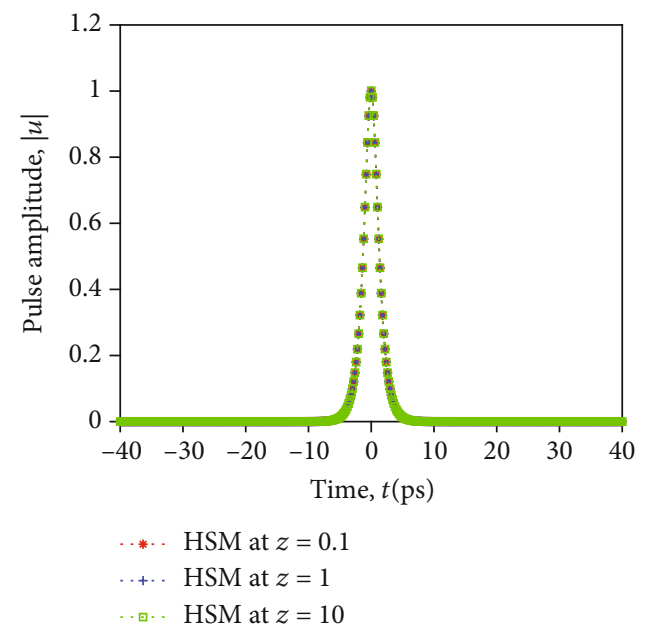

(c)

FIGURE 5: A comparison of the split-step Fourier transform (SSFT) (a), the Fourier pseudospectral method (FPSM) (b), and the Hopscotch method (HSM) (c) approximate solutions at for a lossless optical fiber when the input pulse is a bright soliton at distances $z=0.1,1,10 \mathrm{~km}$, respectively.

$$
i \frac{\partial u(z, t)}{\partial z}=\frac{\beta_{2}}{2} \frac{\partial^{2} u(z, t)}{\partial t^{2}}-\gamma|u(z, t)|^{2} u(z, t)-i \frac{\alpha}{2} u(z, t)
$$

where $u(z, t)$ is the spatial-temporal varying amplitude of the optical pulse, $z$ is the longitudinal coordinate of the fiber, and $t$ is the normalized time with respect to a reference frame that moves with the pulse at a speed equal to the group velocity $v_{g}=1 / \beta_{1}$. This frame is also called the retarded frame, such that $t=\zeta-\left(z / v_{g}\right)$, where $\zeta$ is the present or physical time; both $z$ and $t$ are dimensionless in distance and time, respectively, $\alpha$ is the power attenuation constant, $\gamma$ is the Kerr nonlinear coefficient, and $\beta_{2}$ is the first-order group velocity dispersion (GVD) parameter or the second-order dispersion coefficient $[19,20]$, which can be computed using the following formula:

$$
\beta_{2}=\frac{-\lambda^{2} D}{2 \pi c}
$$

It is significant to highlight that $\lambda$ represents the reference wavelength, $c$ is the speed of light, and $D$ is the fiber dispersion parameter at a given wavelength. In addition to 


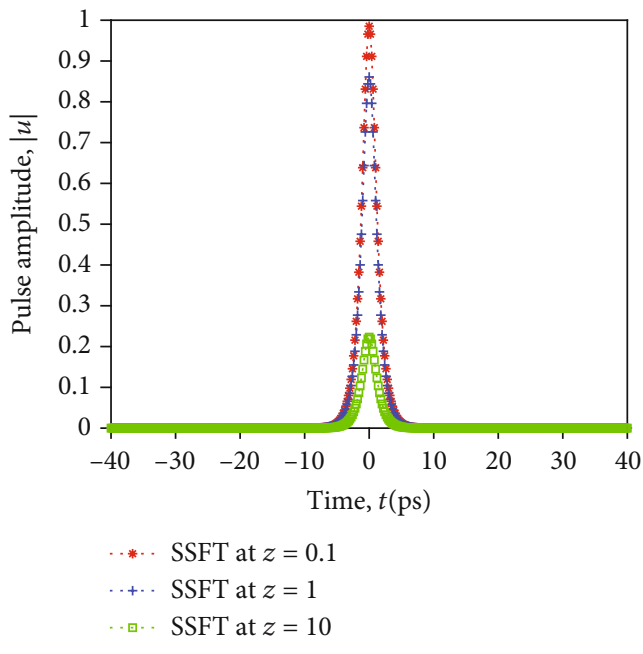

(a)

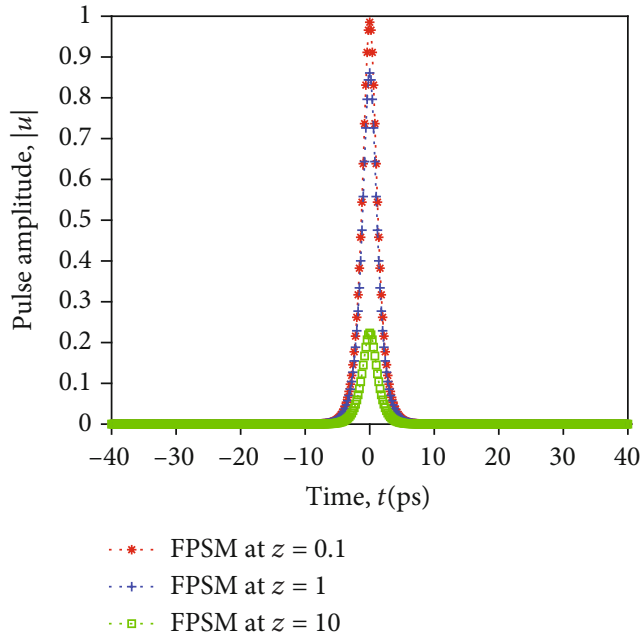

(b)

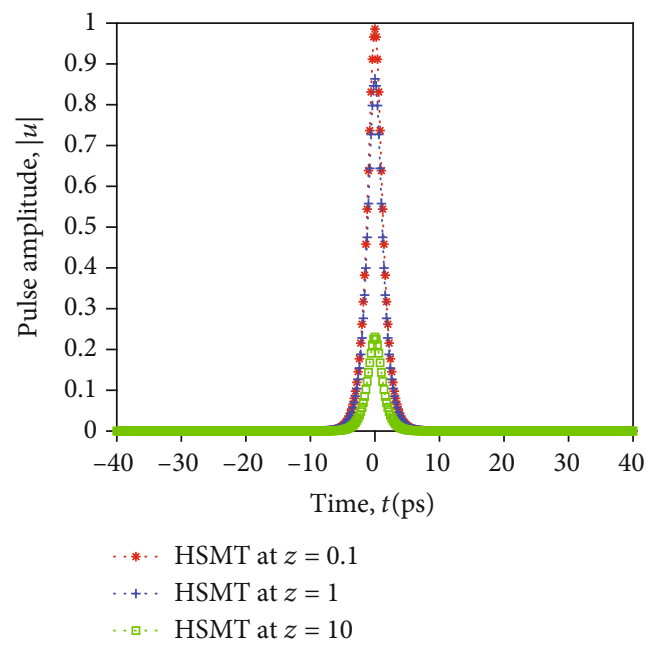

(c)

FIgURE 6: A comparison of the split-step Fourier transform (SSFT) (a), the Fourier pseudospectral method (FPSM) (b), and the Hopscotch method (HSM) (c) approximate solutions at for a lossy optical fiber $\alpha=0.3 \mathrm{~dB} / \mathrm{km}$ when the input pulse is a bright soliton at distances $z$ $=0.1,1,10 \mathrm{~km}$, respectively.

this, the sign of the GVD determines three different regimes as mentioned below:

(i) When $\beta_{2}=0$, the GV is an extremum. Such points are called the zero-dispersion wavelength (ZDW)

(ii) When $\beta_{2}<0$, the GV increases with the frequency, and it is called anomalous dispersion. This regime supports bright solitons

(iii) When $\beta_{2}>0$, the GV decreases with the frequency, and it is called normal dispersion. This regime supports dark solitons

It is obviously noted that the reason of having different velocities for the spectral components of a pulse is the physical behavior of the group velocity $(\mathrm{GV})$, which depends on the frequency. This change in the velocities frequently produces a pulse temporal distortion [31]. Moreover, elaborating the concept of GVD in nonlinear optical fiber is ubiquitously important because of its inevitable effect in shaping the pulse propagation through fiber. When the dispersion is constant, the shape of a well-prepared pulse remains the same, while its propagation through the nonlinear fiber, creating a soliton; the accurate compensation between the nonlinear effects and the linear dispersion in the fiber is the cause of this fixed dispersion.

Likewise, illustrating the fiber loss term is amazingly beneficial as a measure of the power loss during the transmission process inside the fiber. Assuming that the length of the fiber is $L$, the input power is $P_{o}$, and the transmitted power is $P_{t} ; \alpha$ is the attenuation real positive constant. Then, the fiber losses can be calculated using the following formula [6]:

$$
\alpha_{d \sqrt{ } b}=-\frac{10}{L} \log \left(\frac{P_{t}}{P_{o}}\right)=-\frac{10}{L} \log (\exp (-\alpha \mathrm{L}))=4,343 \alpha .
$$




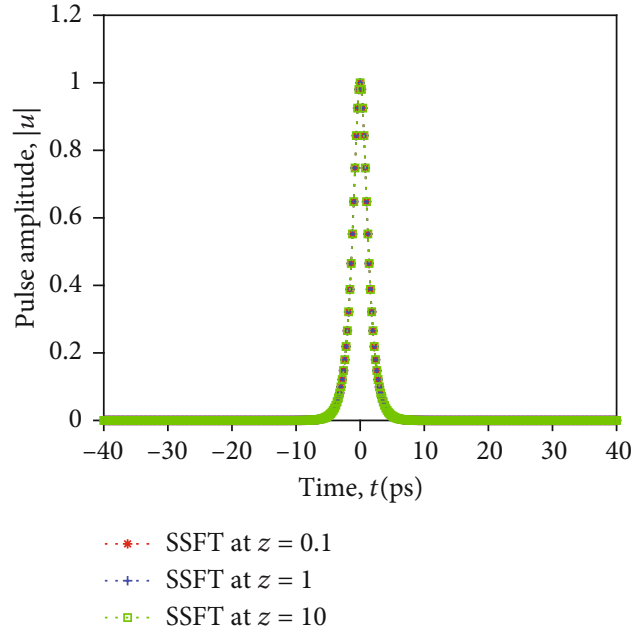

(a)

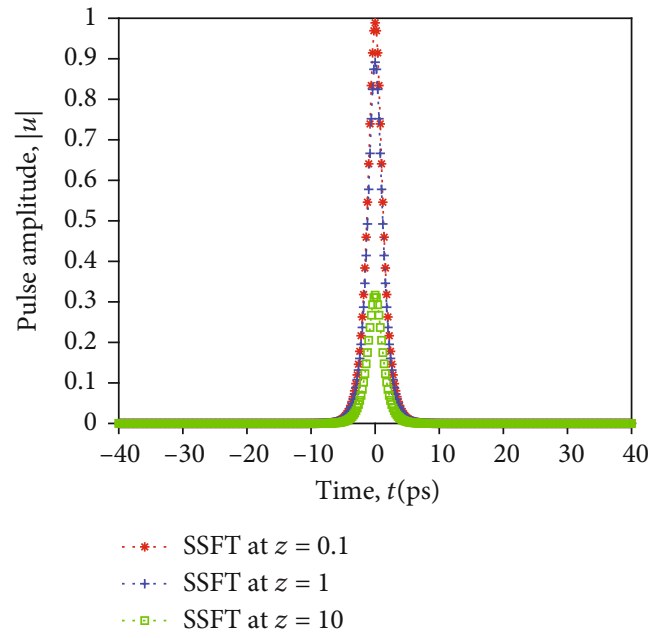

(b)

FIGURE 7: A comparison of the split-step Fourier transform (SSFT) approximate solutions at a higher value of GVD of $\beta_{2}=-20 \mathrm{ps} / \mathrm{km} \mathrm{for} \mathrm{a}$ lossless (a) and lossy optical fiber (b) when the input pulse is a bright soliton at distances $z=0.1,1,10 \mathrm{~km}$, respectively.

Obtaining a more realistic model to simulate and govern the actual wave propagation through the optical fiber requires a generalization of Equation (1). This generalization must include other terms and parameters to demonstrate additional effects. Additionally, the perturbed or generalized nonlinear Schrödinger equation (PNLSE) models this realistic pulses' propagation. If the perturbation does not exist, the PNLSE will be reduced to the canonical nonlinear Schrödinger equation (CNLSE), presented in Equation (8), which has an exact analytical solution that can be obtained using different regimes. On the contrary, when the perturbations take place, its governed equation cannot analytically be solved. It is essential to address different types of optical fiber perturbations, for instance, higher-order and nonlinear dispersion, shock effect, stimulated Raman scattering, self-steepening effects, dissipation, amplification, along with others [32].

The dominance of either the dispersion or the nonlinearity effects might depend on the values of the initial pulse's half-width at half amplitude $T_{0}$ and the initial peak power $P_{o}$ of the incident pulse. Thus, introducing two length scales, which are the dispersion length $L_{D}$ and the nonlinear length $L_{N L}$ is crucially advantageous because the relative magnitude of these two lengths, along with the fiber length $L$, can control the pulses' evolution to some extent. In this regard, we apply a special normalization scale to the time, space, and pulse amplitude as shown below:

$$
\begin{gathered}
T=\frac{t}{T_{0}}, \\
Z=\frac{z}{L}, \\
U(Z, T)=\frac{u(Z, T)}{\sqrt{P_{o}} \exp (-(\alpha Z / 2))} .
\end{gathered}
$$

These transformations yield

$$
\begin{aligned}
i \frac{\partial U(Z, T)}{\partial Z}= & \frac{L}{L_{D}} \frac{\operatorname{sgn}\left(\beta_{2}\right)}{2} \frac{\partial^{2} U(Z, T)}{\partial T^{2}} \\
& -\exp (-\alpha L Z) \frac{L}{L_{N L}}|U(Z, T)|^{2} U(Z, T),
\end{aligned}
$$

where $\operatorname{sgn}\left(\beta_{2}\right)$ is the sign of the $\beta_{2}$ coefficient, $L_{D}=$ $T_{0}{ }^{2} /\left|\beta_{2}\right|$, and $L_{N L}=1 / \gamma P_{o}$, where $L_{D}$ is called the dispersion length that quantifies the pulse broadening per unit length, whereas $L_{N L}$ is defined as the nonlinear length that quantifies the nonlinear phase shift per unit length.

Equation (6) is another formula to represent the NLSE, which might mostly be used in the fabrication process of the fiber optics because this special formalism comprises the fiber real parameters, such as the fiber length, the initial pulse power, and the initial half-width of the input pulse. Nevertheless, this equation may not commonly be employed in the simulations [17, 31].

Moreover, it is fundamental to define the soliton order parameter $\eta$ by the following relation [25]:

$$
\eta^{2}=\frac{L_{D}}{L_{N L}}=\frac{\gamma P_{o} T_{0}^{2}}{\left|\beta_{2}\right|}
$$

when $\eta$ has an integer value; it indicates a soliton pulse solution, either bright or dark, depending on the group velocity dispersion parameter's sign $\beta_{2}$. This integer value manifests that the dispersion and the nonlinear effects compensate each other, achieving a balance that results in an unchanged and undistorted wave during propagation, which is called a soliton.

2.2. Exact Analytical Solution for Solving the CNLSE. To confidently navigate the validation of the proposed numerical 


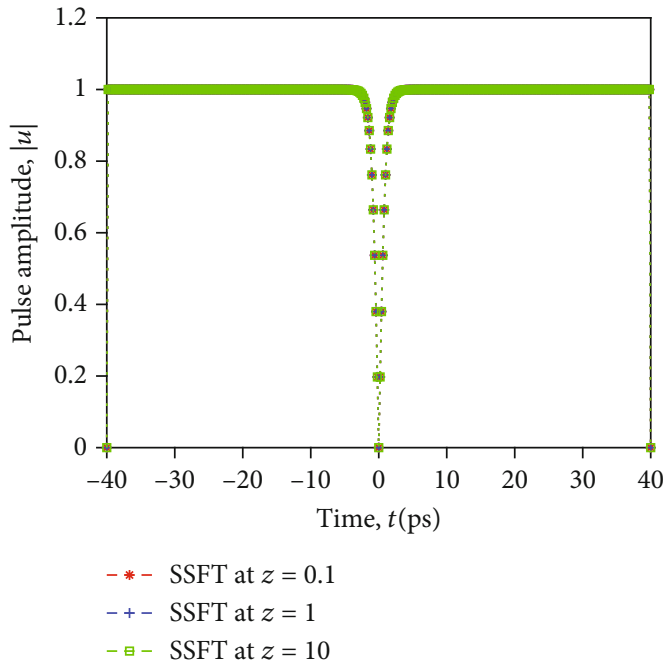

(a)

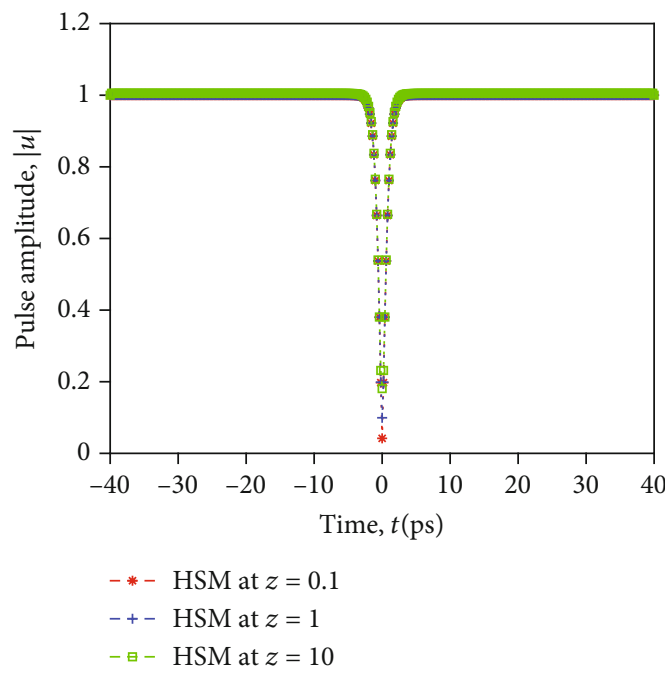

(c)

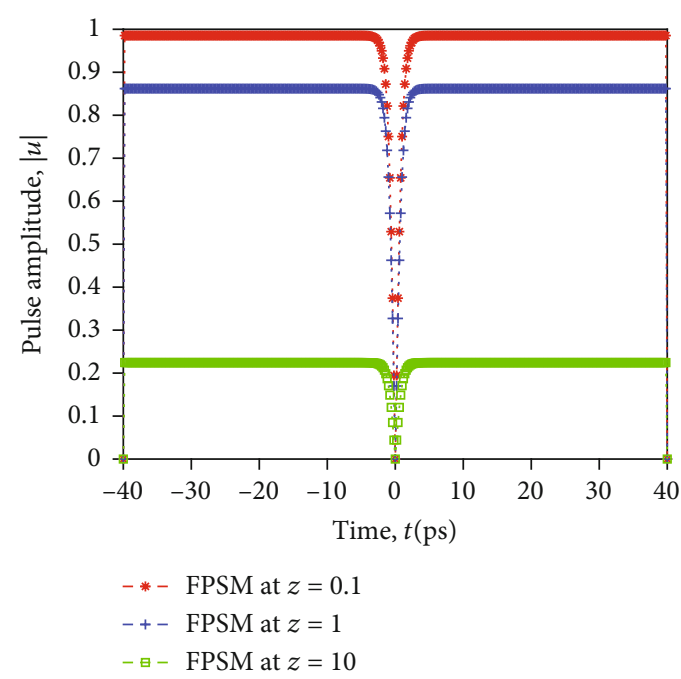

(e)

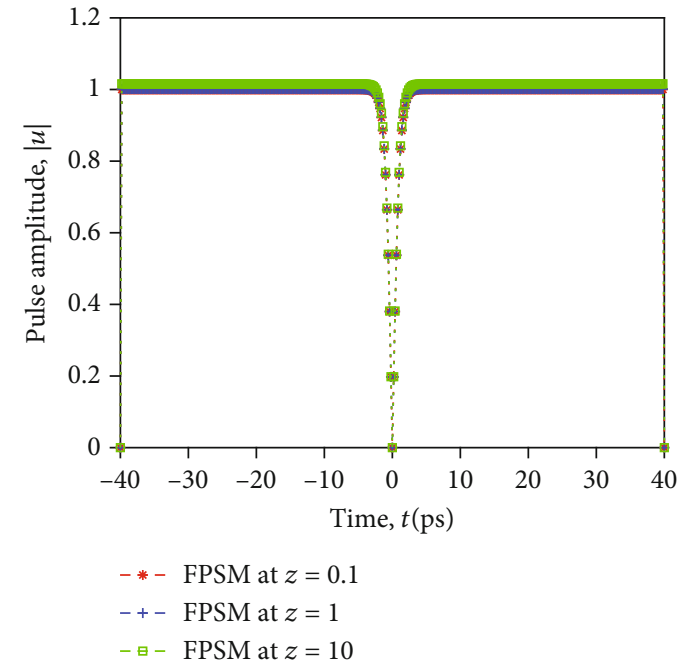

(b)

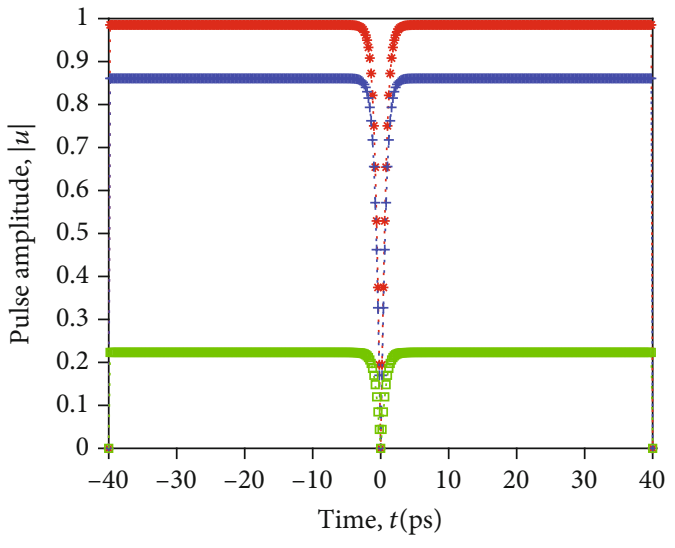

- * - SSFT at $z=0.1$

- + SSFT at $z=1$

$-\because-\operatorname{SSFT}$ at $z=10$

(d)

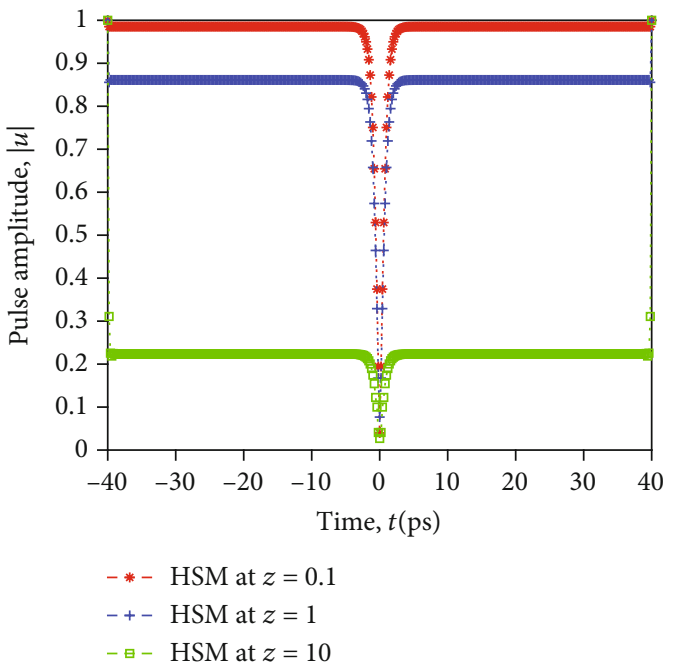

(f)

FIGURE 8: A comparison of the split-step Fourier transform (SSFT), the Fourier pseudospectral method (FPSM), and the Hopscotch method (HSM) approximate solution for a lossless $(\mathrm{a}, \mathrm{b}, \mathrm{c})$ and lossy $(\mathrm{d}, \mathrm{e}, \mathrm{f})$ optical fiber when the input pulse is a dark soliton at distances $z=$ $0.1,1,10 \mathrm{~km}$, respectively. 


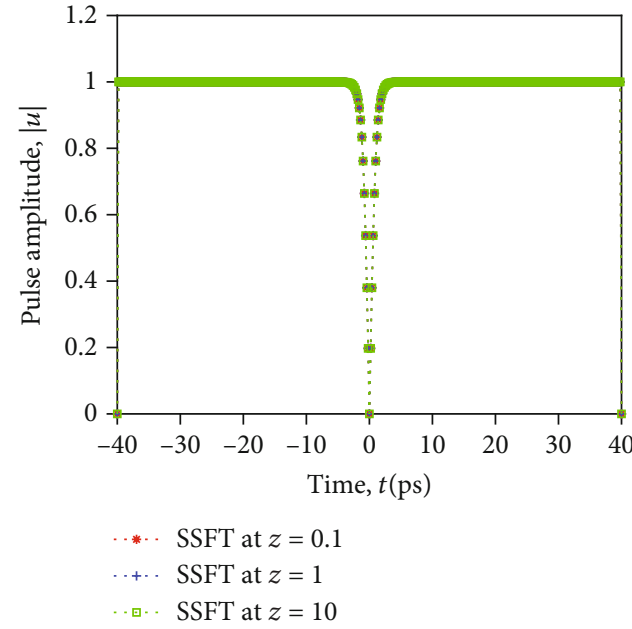

(a)

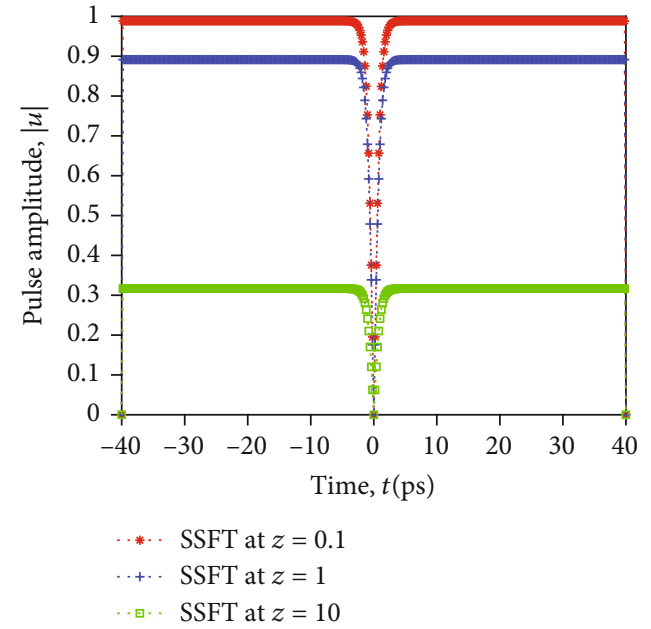

(b)

FIGURE 9: The split-step Fourier transform (SSFT) approximate solution for a lossless (a) and lossy (b) optical fiber at a higher value of GVD of $\beta_{2}=+20 \mathrm{ps}^{2} / \mathrm{km}$ when the input pulse is a dark soliton at distances $z=0.1,1,10 \mathrm{~km}$, respectively.

schemes, a fundamental initiative is to evoke the genuine NLSE along with its exact soliton solution for the comparison purpose. This equation resembles the NLSE under study in this research paper.

However, an interchange between the spatial and temporal variables occurs in each of the first- and second-order derivatives. Beside this, the attenuation term is neglected. Furthermore, it is a nonlinear second-order partial differential equation that is widely used in numerous applications abreast of the optical fiber field. The original NLSE is represented as shown below [33]:

$$
i \frac{\partial u(z, t)}{\partial t}+\frac{\partial^{2} u(z, t)}{\partial z^{2}}+\gamma|u(z, t)|^{2} u(z, t)=0
$$

Similar to Equation (2), the unknown function $u(z, t)$ represents a wave. The second-order derivative $\partial^{2} u(z, t) / \partial$ $z^{2}$ represents the dispersion, while the nonlinear term $\gamma$ $|u(z, t)|^{2} u(z, t)$ represents the nonlinearity of the problem.

In this work, we shall consider and select the single bright soliton solution, shown in Equation (9), also named the envelope solution, among all the other approved exact analytical solutions because of its popularity, along with its simplicity, as it has comprehensively been derived and utilized in a plenty of previously published research articles $[14,26,33,34]$ :

$u(z, t)=(2 \lambda)^{0.5} e^{i\left(0.5 c z+\left(\lambda-0.25 c^{2}\right) t+\Phi o\right)} \operatorname{sech}\left((\lambda)^{0.5}(z-c t-z o)\right)$,

where $z o, \Phi_{o}, c$, and $\lambda$ are the initial position, initial phase, propagation speed, and soliton amplitude, respectively.

2.3. Numerical Approaches for Solving the CNLSE. In this section, three powerful numerical techniques are presented to report the CNLSE, represented in Equation (8). The three methods are the SSFT, FPSM, and HSM [12, 16, 27, 35-40].

2.3.1. The Split-Step Fourier Transform. This method is a straightforward and fast numerical technique that belongs to the pseudospectral family. Moreover, it is unconditionally stable, standing on the split of the NLSE into two subsequent linear and nonlinear partial equations. Moreover, this easy implemented scheme has a unique significance because it represents the effects of the dispersion and nonlinearity separately in this problem.

Rearranging the terms of Equation (8), on the form of $\partial_{t} u(z, t)=(\mathrm{L}+\mathrm{N}) u(z, t)$, to isolate the linear and nonlinear terms yields

$$
\begin{gathered}
i \partial_{t} u(z, t)=-\partial_{z z}^{2} u(z, t)-\gamma|u(z, t)|^{2} u(z, t), \\
u(z, 0)=u_{0}(z)
\end{gathered}
$$

Assuming that the linear operator is $L=i \partial_{z z}^{2}$ and the nonlinear operator is $N=\gamma i|u(z, t)|^{2}$. Then, we split it into two parts to solve the problem as follows.

Part one, the nonlinear step is introduced as: $\partial_{t} u(z, t)$ $=N u(z, t)$, where $N=\gamma i|u(z, t+\tau)|^{2} \approx \gamma i|u(z, t)|^{2}$. Hence, the analytical solution will be given as shown below:

$$
u(z, t+\tau)=\exp (i \tau N) u(z, t)=\exp \left(i \tau \gamma|u(z, t)|^{2}\right) u(z, t)
$$

Part two, the linear step is introduced as $i \partial_{t} u(z, t)=\mathrm{L} u$ $(z, t)$. We apply Fourier transform, to both sides to convert the PDE into an ODE in the frequency domain to facilitate its solution, as follows:

$$
\partial_{t} \widehat{u}=-i k^{2} \widehat{u}
$$




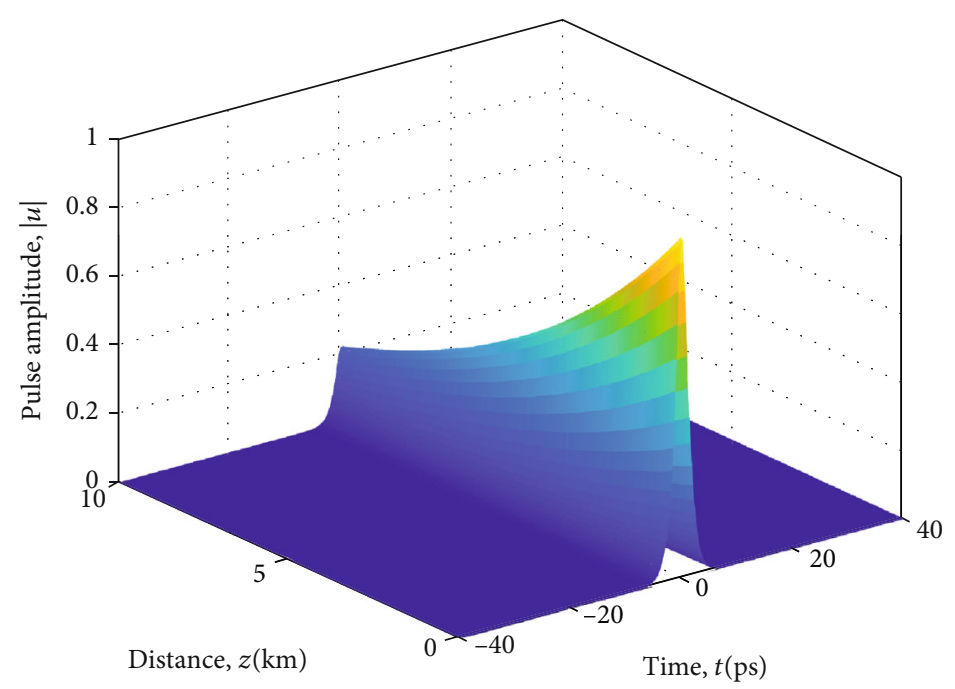

(a)

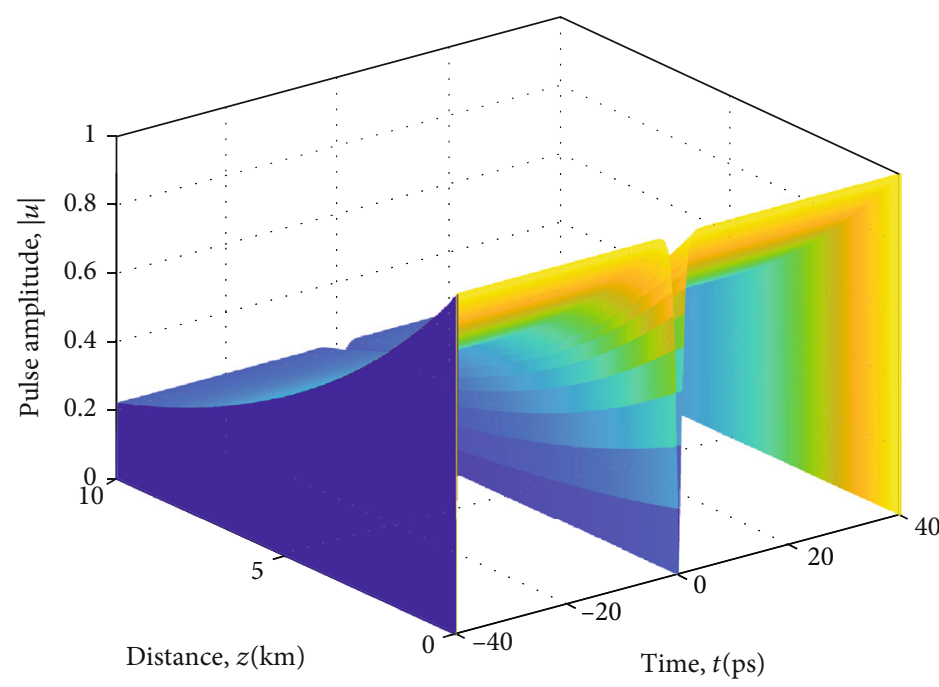

(b)

FIGURE 10: Three-dimension graphs of the split-step Fourier transform (SSFT) approximate solution for a lossy optical fiber $\alpha=0.3 \mathrm{~dB} / \mathrm{km}$ when the input pulse is a bright soliton (a) and a black dark soliton (b), respectively, at a distance $z=10 \mathrm{~km}$.

Equation (14) demonstrates the analytical solution of the previous equation but computed in the frequency domain:

$$
\widehat{u}(z, t+\tau)=\exp \left(-i k^{2} \tau\right) \cdot \widehat{u}(z, t) .
$$

Eventually, we apply the inverse Fourier transform to both sides to obtain the final equation represented below:

$$
u(z, t+\tau)=F^{-1}\left(\exp \left(-i k^{2} \tau\right) \cdot F\left(\exp \left(i \tau \gamma|u(z, t)|^{2}\right) u(z, t)\right)\right) \text {. }
$$

2.3.2. The Fourier Pseudospectral Method. This approach is deemed to be a member of the pseudospectral family as well, and it can only be applied to the periodic functions over the interval $x \in[-P, P]$. Its key pillar stands on implementing Fourier transform for the space second-order derivative, while discretizing the first-order time derivative using an appropriate finite difference relation.

In the first stage, we replace the temporal first derivative by the following difference relation:

$$
\frac{\partial u}{\partial t}=\frac{u(z, t+\tau)-u(z, t)}{\tau} .
$$

In the second stage, we substitute the above relation into Equation (8) to get the following equation:

$$
i \frac{u(z, t+\tau)-u(z, t)}{\tau}=-F^{-1}\left(i^{2} k^{2} \frac{\pi^{2}}{P^{2}} F(u)\right)-\gamma|u(z, t)|^{2} u(z, t),
$$


TABLE 4: A comparison of the pulse powers using the split-step Fourier transform (SSFT), the Fourier pseudospectral method (FPSM), and the Hopscotch scheme (HSM), at a distance $z=20 \mathrm{~km}$, computed at different attenuation constants values $\alpha$ from 0.1 to $0.45 \mathrm{~dB} / \mathrm{km}$.

\begin{tabular}{lcccccc}
\hline$\alpha(\mathrm{dB} / \mathrm{km})$ & Power $(\mathrm{w})-\mathrm{SSFT}$ & CPU time $(\mathrm{s})$ & Power $(\mathrm{w})$-FPSM & CPU time $(\mathrm{s})$ & Power $(\mathrm{w})$-HSM & CPU time $(\mathrm{s})$ \\
\hline 0.1 & $1.3534 e-01$ & 1.1251 & $1.3579 e-01$ & 1.2936 & $1.4115 e-01$ & 2.9371 \\
0.15 & $4.9787 e-02$ & 1.1236 & $5.0051 e-02$ & 1.2766 & $5.3130 e-02$ & 2.9356 \\
0.2 & $1.8316 e-02$ & 1.1046 & $1.8436 e-02$ & 1.296 & $2.0022 e-02$ & $7.5516 e-03$ \\
0.25 & $6.7379 e-03$ & 1.1287 & $6.7884 e-03$ & 1.3048 & 2.85 \\
0.3 & $2.4788 e-03$ & 1.108 & $2.4988 e-03$ & 1.3188 & $1.8516 e-03$ & 2.9684 \\
0.35 & $9.1188 e-04$ & 1.1347 & $9.1961 e-04$ & 1.2802 & $3.0824 e-03$ & 3.0699 \\
0.4 & $3.3546 e-04$ & 1.1467 & $3.3838 e-04$ & 1.2865 & $1.569 e-04$ & 3.1696 \\
0.45 & $1.2341 e-04$ & 1.1515 & $1.2450 e-04$ & 1.2792 & 3.0975 \\
\hline
\end{tabular}

TABLE 5: A comparison of the pulse powers using the split-step Fourier transform (SSFT), the Fourier pseudospectral method (FPSM), and the Hopscotch method (HSM), at an attenuation constant $\alpha=0.2 \mathrm{~dB} / \mathrm{km}$, computed at different distances $z$ from 1 to $60 \mathrm{~km}$.

\begin{tabular}{lcccccc}
\hline$z(\mathrm{~km})$ & Power $(\mathrm{w})-$ SSFT & CPU time $(\mathrm{s})$ & Power $(\mathrm{w})$-FPSM & CPU time $(\mathrm{s})$ & Power $(\mathrm{w})$-HSM & CPU time $(\mathrm{s})$ \\
\hline 1 & $8.1873 e-01$ & 0.066308 & $8.1890 e-01$ & 0.07973 & $8.2207 e-01$ & 0.20215 \\
5 & $3.6788 e-01$ & 0.31196 & $3.6802 e-01$ & 0.35977 & $3.7538 e-01$ & 0.82056 \\
10 & $1.3534 e-01$ & 0.55666 & $1.3557 e-01$ & 0.65937 & $1.4107 e-01$ & $2.0021 e-02$ \\
20 & $1.8316 e-02$ & 1.1563 & $1.8436 e-02$ & 1.2707 & $2.8636 e-03$ & 4.0384 \\
30 & $2.4788 e-03$ & 1.6731 & $2.5091 e-03$ & 2.0559 & $2.1551 e-04$ & 5.8678 \\
40 & $3.3546 e-04$ & 2.2148 & $3.4151 e-04$ & 2.5027 & $6.3139 e-05$ & 7.2459 \\
50 & $4.5400 e-05$ & 2.7437 & $4.6481 e-05$ & 3.1548 & $3.3284 e-05$ \\
60 & $6.1442 e-06$ & 3.3364 & $6.3267 e-06$ & 3.7705 & 9.1225 \\
\hline
\end{tabular}

$u(z, t+\tau)-u(z, t)=\tau i F^{-1}\left(i^{2} k^{2} \frac{\pi^{2}}{P^{2}} F(u)\right)+i \tau \gamma|u(z, t)|^{2} u(z, t)$

$u(z, t+\tau)=u(z, t)-\tau i F^{-1}\left(k^{2} \frac{\pi^{2}}{P^{2}} F(u)\right)+i \tau \gamma|u(z, t)|^{2} u(z, t)$.

The solution exhibited in Equation (18) is only stable for values of $\tau /(h)^{2}<1 / \pi^{2}$.

Despite this, applying the Fornberg and Whitham principles yields an unconditionally stable solution, as follows:

$$
\begin{aligned}
u(z, t+\tau)= & u(z, t)-\mathrm{iF}^{-1}\left(\sin \left(k^{2} \frac{\pi^{2}}{P^{2}} \tau\right) F(u(z, t))\right) \\
& +i \tau \gamma|u(z, t)|^{2} u(z, t)
\end{aligned}
$$

2.3.3. The Hopscotch Method. This scheme is a fast explicit finite difference method, which operates on replacing the nonlinear term by an average formula computed at the row $j$, as shown in Equation (20). Besides, it is also an unconditionally stable approach with a truncation error of
$O\left(h^{2}\right)+O\left(\tau^{2}\right)$ [27]; thus, the order of convergence is 2 in both time and distance.

$$
(u)_{i j}=\left(\frac{\left|u_{i-1, j}\right|^{2} * u_{i-1, j}+\left|u_{i+1, j}\right|^{2} u_{i+1, j}}{2}\right) .
$$

We substitute the previous equation and the other appropriate difference relations in Equation (8), subject to the boundary and initial conditions, which yields

$$
\begin{aligned}
i \frac{u_{i, j+1}-u_{i, j}}{\tau}+ & \frac{u_{i+1, j}+u_{i-1, j}-2 u_{i, j}}{h^{2}} \\
& +\gamma \frac{\left|u_{i-1, j}\right|^{2} * u_{i-1, j}+\left|u_{i+1, j}\right|^{2} u_{i+1, j}}{2}=0,
\end{aligned}
$$

$$
\begin{aligned}
u_{i, j+1}= & u_{i, j}+i \tau \frac{u_{i+1, j}+u_{i-1, j}-2 u_{i, j}}{h^{2}} \\
& +i \gamma \frac{\tau}{2}\left(\left|u_{i-1, j}\right|^{2} * u_{i-1, j}+\left|u_{i+1, j}\right|^{2} u_{i+1, j}\right) .
\end{aligned}
$$




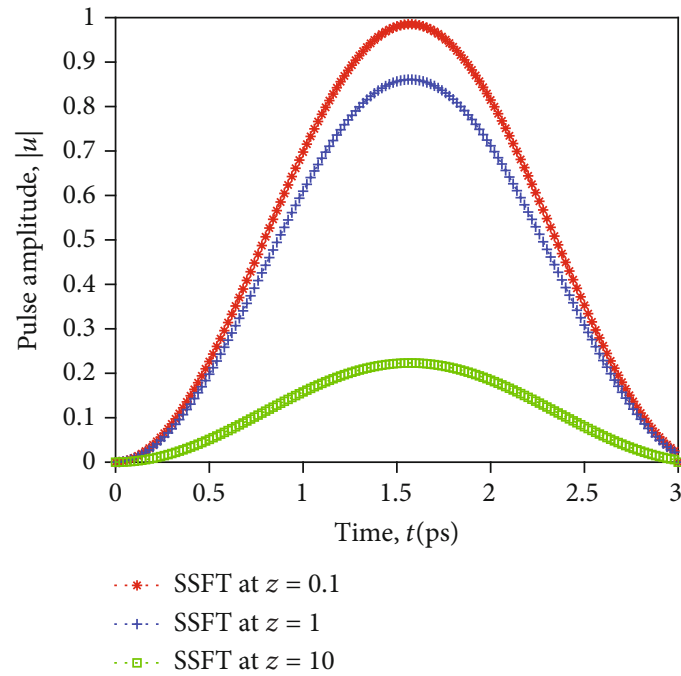

(a)

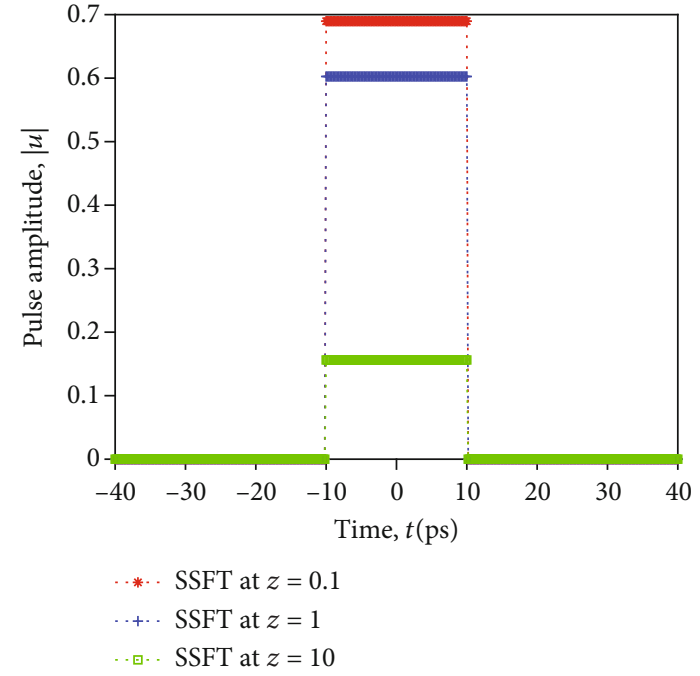

(b)

FIgURe 11: The split-step Fourier transform (SSFT) approximate solution for a lossy optical fiber $\alpha=0.3 \mathrm{~dB} / \mathrm{km}$ when the input pulse is a sine squared function (a) and a rectangular pulse (b) at distances $z=0.1,1,10 \mathrm{~km}$, respectively.

Therefore, the final explicit formula is given by Equation (22), where $\lambda=\tau / h^{2}$,

$$
\begin{aligned}
u_{i, j+1}= & (1-2 i \lambda) u_{i, j}+i \lambda\left(u_{i+1, j}+u_{i-1, j}\right) \\
& +i \gamma \frac{\tau}{2}\left(\left|u_{i-1, j}\right|^{2} * u_{i-1, j}+\left|u_{i+1, j}\right|^{2} u_{i+1, j}\right) .
\end{aligned}
$$

\section{Modified Numerical Approaches for Solving the Lossy Fiber Optics}

In this section, two main actions were implemented to obtain the modified versions. First, we have successfully incorporated the loss term in the three suggested numerical schemes, which are the SSFT, FSSM, and HSM. This unique insertion is utterly essential in the simulation process because it guarantees preserving a high accuracy level for the obtained approximate solutions that should effectively be employed to model the propagation of the solitons. Second, an interchange has taken place between the temporal and spatial variables to cope with the requirements of the problem, under study in this article. As a result, extended versions of each of the three proposed numerical techniques are derived to solve the 1D NLSE, comprising the attenuation term, presented in Equation (1) or (2). Thereafter, comparing their resulted approximate solutions to corroborate their reliability and creditability, here, the exact analytical solution is an inadequate trend because the exact solution cannot smoothly be obtained for this kind of equation, especially after inserting the term that is responsible for the fiber losses. Roughly speaking, these modifications seek an insightful approximation of the attenuation amount to eradicate its undesired effects by using compensating amplifiers, which guarantees the healthy delivery of the transmitted signals.
3.1. The Extended Split-Step Fourier Transform. Starting with the NLSE presented in Equation (1) and rewriting it as shown below:

$$
\partial_{z} u(z, t)=(\mathrm{L}+\mathrm{N}) u(z, t) .
$$

While applying the initial condition $u(0, \mathrm{t})=u_{0}(t)$. Then, define the time independent linear operator as $L=-i$ $\left(\beta_{2} / 2\right) \partial_{t t}^{2}-(\alpha / 2)$, and the nonlinear operator as $N=i \gamma$ $|u(z, t)|^{2}$.

First, the analytical solution of the nonlinear part will be given by the following equation:

$$
u(z+h, t)=\exp \left(i h \gamma|u(z, t)|^{2}\right) u(z, t) .
$$

Second, the analytical solution of the linear part can be computed in the frequency domain, using the following relation:

$$
\widehat{u}(z+h, t)=\exp \left(i \frac{\beta_{2}}{2} k^{2} h\right) \cdot \exp \left(-\frac{\alpha}{2} h\right) \cdot \widehat{u}(z, t) .
$$

Then, plugging Equation (24) into the previous equation:

$$
\begin{aligned}
\widehat{u}(z+h, t)= & \exp \left(i \frac{\beta_{2}}{2} k^{2} h\right) \cdot \exp \left(-\frac{\alpha}{2} h\right) \cdot F \\
& \cdot\left(\exp \left(i h \gamma|u(z, t)|^{2}\right) u(z, t)\right) .
\end{aligned}
$$

Hence, the final equation can be written as

$$
\begin{aligned}
u(z+h, t)= & F^{-1}\left(\exp \left(i \frac{\beta_{2}}{2} k^{2} h\right) \cdot \exp \left(-\frac{\alpha}{2} h\right) \cdot F\right. \\
& \left.\cdot\left(\exp \left(i h \gamma|u(z, t)|^{2}\right) u(z, t)\right)\right) .
\end{aligned}
$$


3.2. The Extended Fourier Pseudospectral Method. Commencing with the NLSE over the interval $t \in[-P, P]$. First, replacing the spatial first-order derivative by the following difference relation:

$$
\frac{\partial u}{\partial z}=\frac{u(z+h, t)-u(z, t)}{h}
$$

Second, substituting the above relation into Equation (1) to get the following equation:

$$
\begin{aligned}
\frac{u(z+h, t)-u(z, t)}{h}= & -i \frac{\beta_{2}}{2} F^{-1}\left(i^{2} k^{2} \frac{\pi^{2}}{P^{2}} F(u)\right) \\
& +i \gamma|u(z, t)|^{2} u(z, t)-\frac{\alpha}{2} u(z, t), \\
u(z+h, t)-u(z, t)= & -i \frac{\beta_{2}}{2} h F^{-1}\left(i^{2} k^{2} \frac{\pi^{2}}{P^{2}} F(u)\right) \\
& +i \gamma h|u(z, t)|^{2} u(z, t)-\frac{\alpha}{2} h u(z, t) .
\end{aligned}
$$

Then, substituting the difference relation in Equation (28), the initial condition $u(0, \mathrm{t})=u_{0}(t)$, and the Fourier transform of the second derivative term into Equation (1) to obtain the following equation:

$$
\begin{aligned}
u(z+h, t)= & u(z, t)+h \frac{\beta_{2}}{2} \mathrm{iF}^{-1}\left(k^{2} \frac{\pi^{2}}{P^{2}} F(u)\right) \\
& +i \gamma h\left|u(z, t)^{2}\right| u(z, t)-\frac{\alpha}{2} h u(z, t) .
\end{aligned}
$$

Equation (30) is only stable for values of $h / \tau^{2}<1 / \pi^{2}$.

Despite this, making a modification in the previous equation by using the Fornberg and Whitham principles [27] yields an unconditionally stable solution, presented

$$
\begin{aligned}
u(z+h, t)= & u(z, t)+\frac{\beta_{2}}{2} \mathrm{iF}^{-1}\left(\sin \left(k^{2} \frac{\pi^{2}}{P^{2}} h\right) F(u(z, t))\right) \\
& +i \gamma h\left|u(z, t)^{2}\right| u(z, t)-\frac{\alpha}{2} h u(z, t) .
\end{aligned}
$$

3.3. The Extended Hopscotch Method. To kick off, we replace the nonlinear term by an average formula computed at the column $i$ as illustrated below:

$$
(u)_{i j}=\left(\frac{\left|u_{i, j-1}\right|^{2} * u_{i, j-1}+\left|u_{i, j+1}\right|^{2} u_{i, j+1}}{2}\right) .
$$

Substituting the appropriate FDM average and difference relations in Equation (1), subject to the initial and boundary conditions, to obtain

$$
\begin{aligned}
\frac{u_{i+1, j}-u_{i, j}}{h}+ & \frac{u_{i, j+1}+u_{i, j-1}-2 u_{i, j}}{\tau^{2}} \\
& +i \gamma \frac{\left|u_{i, j-1}\right|^{2} * u_{i, j-1}+\left|u_{i, j+1}\right|^{2} u_{i, j+1}}{2} \\
& -\frac{\alpha}{2} h\left(\frac{u_{i, j-1}+u_{i, j+1}}{2}\right)=0, \\
u_{i+1, j}= & u_{i, j}-i \frac{\beta_{2}}{2} h\left(\frac{u_{i, j+1}+u_{i, j-1}-2 u_{i, j}}{\tau^{2}}\right) \\
& +i \gamma \frac{h}{2}\left(\left|u_{i, j-1}\right|^{2} * u_{i, j-1}+\left|u_{i, j+1}\right|^{2} u_{i, j+1}\right) .
\end{aligned}
$$

The final explicit formula for this unconditionally stable approach is given by

$$
\begin{aligned}
u_{i+1, j}= & \left(1+i \beta_{2} \lambda\right) u_{i, j}-i \frac{\beta_{2}}{2} \lambda\left(u_{i, j+1}+u_{i, j-1}\right) \\
& +i \gamma \frac{h}{2}\left(\left|u_{i, j-1}\right|^{2} * u_{i, j-1}+\left|u_{i, j+1}\right|^{2} u_{i, j+1}\right) \\
& -\frac{\alpha}{4} h\left(u_{i, j-1}+u_{i, j+1}\right),
\end{aligned}
$$

where $\lambda=h / \tau^{2}$.

\section{The Error and Convergence Discussion}

The convergent statement states that the numerical solutions collapse onto the exact analytical solution, when the limit $\tau$ $\longrightarrow 0$; hence, the error reaches zero accordingly at all time indices. In other words, if we shrink the temporal step towards zero smaller and smaller, the absolute error, which is the difference between the exact and approximate solution, will go smaller and smaller as well. The convergence of the three numerical schemes is verified numerically in the fifth section under systematic temporal mesh refinement [41].

\section{Numerical Results}

Here, our strategy is to first investigate the behavior of our original proposed numerical approaches against the exact analytical solution for the CNLSE, which models the pulse propagation in a lossless optical fiber. Then, the extended versions of these numerical techniques are intensively employed to solve the PNLSE, associated with the fiber loss term.

5.1. Numerical Tests for the CNLSE. In this section, plenty of designated numerical examples are conducted, as recently followed elsewhere [42-47], to examine how efficient, fast, and accurate the proposed numerical techniques are, especially when compared with the exact analytical solution. Particularly, MATLAB software was used to run these tests, which were performed to measure the accuracy among 
various numerical approaches, estimate the error, and decide on the most reliable and fastest approach for solving the $1 \mathrm{D}$ NLSE. In all the proposed methods, the NLSE is discretely solved at different values of time and space [28]. For the numerical experiment, we discretize the space domain $x$ from -40 to 40 , setting the parameters, in Equation (9), $\gamma$ nad $\lambda$, to one unit and $c$, xo, and $\Phi_{o}$ to zero, respectively. This leads to the hyperbolic secant initial condition [37]:

$$
u(z, 0)=(2)^{0.5} e^{i(0.5 z)} \operatorname{sech}(z)
$$

Associated with the zero boundary conditions is shown below:

$$
\begin{gathered}
u(L, t)=0, \\
u(-L, t)=0,
\end{gathered}
$$

occurring at $z=-L, L$, and for $t \geq 0$.

Our simulation strategy stands on employing various spatial steps $h$ when the other parameters are fixed, and subsequently, adapting various temporal steps $\tau$ while not changing the rest of the parameters. These steps sizes are dimensionless, and their values determine the accuracy of the experiment. In particular, the smaller their values, the more precise the approximate numerical solution becomes. Figure 2 demonstrates the graph of the exact bright one soliton solution in three dimensions to provide a rigorous focus during the comparison process on the actual shape of this pulse.

First, by using numerous space steps at a time step $\tau=$ 0.001 computed at time $t=20$, we have compared the exact solution, previously presented in Equation (9), and the three suggested methods, which are the split-step Fourier method, Fourier pseudospectral method, and Hopscotch method, in the graphs presented in Figure 3. Second, although the same process has been repeated, the values of the used time step have been changed instead of the space step, which sustained a constant value of 0.2 , computed at the same time $t=20$. The graphs are presented in Figure 4.

Eventually, drawing a comparison to estimate the sum of squares error (SSE) [48] of all the mentioned numerical techniques and their exact solution, shown in Table 1, at the selected spatial step $h=0.2$, temporal step $\tau=0.001$, and space domain $x$ from -40 to 40 , while selecting a domain of time values from 35 to 105 . In specific, the SSE is computed by $\sum_{i=1}^{n}\left(u_{i}-u_{e x}\right)^{2}$, where $u_{i}$ is the discrete numerical approximate solution over the predefined domain $x$ and $u_{\text {ex }}$ is the discrete exact analytical solution over the same predefined spatial domain at a specific time value. As endorsed by Figures 3 and 4, the three proposed approaches are accuracy adjustable. The smaller the temporal and spatial steps sizes, the more efficacy they achieve. Besides, as advocated by Table 1, the SSFT approach exhibits the smallest sum of squares error over the other demonstrated schemes. Precisely, the errors are approximately in terms of $10 e-21$ when the time step is $10 e-3$, which substantiate that this method almost renders the exact solution itself. However, we can achieve less computational time by adjusting a higher value of temporal step size, in a trade off with the accuracy of the scheme that will be decreased accordingly, as demonstrated in Tables 2 and 3. Additionally, it is obviously noticed from Tables 1-3, using a fixed space step size while employing different values of time steps $\tau=0.008, \tau=$ 0.001 , and $\tau=0.0001$, that the obtained error diminishes when the temporal step size value decreases in an attempt to approach zero, which advocates the convergence behavior of our three proposed schemes [41].

5.2. Numerical Tests for the PNLSE. Here, we shall divert our attention to the PNLSE presented in Equation (1), in which the fiber loss term deliberately appears so that we intensively investigate this perturbation effect on the shape of the transmitted pulse.

In order to examine such an effect, our numerical experiments have been performed on both types of solitons solutions, single bright and dark solitons.

To perform these tests, we shall use an initial bright pulse soliton of [25]:

$$
u(0, t)=\sqrt{P_{o}} \operatorname{sech}\left(\frac{t}{T_{0}}\right)
$$

On the other hand, using an initial dark pulse soliton of

$$
u(0, t)=\sqrt{P_{o}}\left(\frac{1}{B^{2}}-\operatorname{sech}^{2}\left(\mathrm{~B} \frac{t}{T_{0}}\right)\right)^{0.5}
$$

Setting $P_{o}$ and $T_{o}$ to one unit for both initial conditions. In Equation (38), $B$ is called the darkness coefficient. In general, the value of this coefficient varies between zero and one $0<B \leq 1$.

Opting for $B=1$, this soliton is called a "black soliton." In contrast, a grey soliton is produced, for any other value of $B$ [4]. Since the exact solution cannot be obtained for these kinds of equations, the three proposed approaches were examined for a lossy optical fiber. Generally, the simulation process is divided into three fundamental consecutive stages.

In the first stage, our simulation is focused on the anomalous dispersion, generating bright solitons for an optical fiber of length $L=10 \mathrm{~km}$. Moreover, this assessment was performed, adhering to the following parameters $\beta_{2}=-1$ $\mathrm{ps}^{2} / \mathrm{km}, \gamma=1 \mathrm{~W}^{-1} \cdot \mathrm{km}^{-1}$, when $\alpha=0 \mathrm{~dB} / \mathrm{km}$ followed by $\alpha=$ $0.3 \mathrm{~dB} / \mathrm{km}$. The graphs, representing the pulse at different distances $z=0.1,1,10 \mathrm{~km}$, are shown in Figures 5 and 6 , respectively. Likewise, repeating the previous experiment for the same fiber but with different parameters' values and higher GVD such that $\beta_{2}=-20 \mathrm{ps}^{2} / \mathrm{km}, \gamma=2.5 \mathrm{~W}^{-1} \mathrm{~km}^{-1}$, and $\alpha=0 \mathrm{~dB} / \mathrm{km}$. Since the split-step Fourier transform scheme is the only method that has endured under this assessment, the graph of its approximate solution is shown in Figure 7(a). Subsequently, the experiment was conducted for a lossy optical fiber at the same length and parameters, when $\alpha=0.23 \mathrm{~dB} / \mathrm{km}$. Figure 7(b) exhibits the pulses' shapes at different distances using the split-step Fourier transform as well. 
In the second stage of this simulation, our goal is to test the normal dispersion, introducing dark solitons pulses. For this purpose, the approach was examined on the same length of optical fiber, when $\beta_{2}=+1 \mathrm{ps}^{2} / \mathrm{km}$, and $\gamma=1 \mathrm{~W}^{-1} \mathrm{~km}^{-1}$, and for both values of attenuation constant $\alpha=0$ and $0.3 \mathrm{~dB} / \mathrm{km}$. The three numerical schemes were tested, and their results are illustrated in Figure 8. Similarly, we have repeated the previous experiment but with other parameters' values such that $\beta_{2}=+20 \mathrm{ps}^{2} / \mathrm{km}, \gamma=2.5 \mathrm{~W}^{-1} \mathrm{~km}^{-1}$, and for both $\alpha=0$ and $0.23 \mathrm{~dB} / \mathrm{km}$. The results, by using the splitstep Fourier transform, are presented in Figure 9. In Figures 10(a) and 10(b), we have plotted the split-step Fourier transform numerical solution in 3D for a lossy optical fiber $\alpha=0.3 \mathrm{~dB} / \mathrm{km}$ when the input pulse is a single bright and dark soliton, respectively, at a distance $z=10 \mathrm{~km}$.

For a single bright soliton, a comparison is conducted in Table 4, using the three proposed numerical schemes, to present the variation of the pulse power values at a distance $z=20 \mathrm{~km}$, computed for different attenuation constants, while Table 5 demonstrates the attenuation effect on the transmitted pulse power, using the same numerical approaches, when the attenuation constant is $\alpha=0.2 \mathrm{~dB} / \mathrm{km}$ but over different distances $z$ from 1 to $60 \mathrm{~km}$. The final stage is dedicated to applying the same test but for various initial conditions, associated with different input pulses, which cannot be supported with the exact solution that is only valid for some special initial pulses. This means other signals rather than the bright and dark solitons, such as the sine squared function, which is a periodic travelling wave, and the rectangular pulse in the presence of attenuation. Figure 11 presents the outcome of this experiment, by using the split-step Fourier transform to approximate the solution.

As evident by all the obtained numerical results, our suggested approach, which is the extended split-step Fourier transform, has a leading behavior in modeling the soliton propagation through a lossy optical fiber, especially when compared to the other proposed numerical approaches. Based on the previously obtained findings, Figures 5 and $8(a)-8(c)$ reinforced that the input signal has retained its shape and amplitude while propagation, in the absence of the losses, creating a soliton. On the contrary, when the fiber losses existed, the transmitted pulse has suffered from attenuation, which can be noticed as a decrease in the amplitude and power of the pulse [49], as supported by Figures 6 and $8(\mathrm{~d})-8(\mathrm{f})$, whereas Figure 10 bolstered that this amplitude degradation gradually occurs in an exponential form over the propagation distance. Hence, amplifiers are widely used to overcome such a perturbed effect. Moreover, when the GVD parameter maintained a value less than 4 , the three proposed schemes have provided a reliable solution for this problem, as referred in Figures 5, 6, and 8. Nevertheless, when this parameter was assigned a value greater than or equal to five, the extended split-step Fourier transform was the only approach that has provided reasonable behavior under this experiment and achieved the best accuracy among the other proposed techniques, as explained in Figures 7 and 9. Eventually, Table 4 endorsed that increasing the fiber losses has inversely decreased the transmitted pulse power. Meanwhile, transmitting the pulse through longer propagation distance in a lossy optical fiber has conversely attenuated the power of the initial pulse, as illustrated by Table 5.

Despite the three extended numerical approaches have achieved slightly close results, as clearly shown in Tables 4 and 5, our superior approach, the extended SSFT, has corroborated the fastest performance, through achieving the least elapsed time among the other extended versions of the other demonstrated techniques.

\section{Conclusion}

To recapitulate, although the pulse propagation through a lossy optical fiber is modeled by the well-known 1D NLSE, the exact solution of this equation cannot invariably be reached, especially after comprising the attenuation term. Thus, an extended version of the SSFT approach was successfully developed in this paper by including the fiber loss effects to accurately obtain an approximate numerical solution that simulates this notable phenomenon. This precise inclusion is extremely crucial because these losses, associated with any realistic optical fiber, cannot be ignored due to their inevitable repercussions in decreasing the amplitude and power of the soliton during propagation over the transmission path. Additionally, to advocate the efficacy and accuracy of our proposed approach, two other extensions of numerical schemes were introduced, named the FPSM and HSM, and assessed by drawing a plethora of graphs and tables for the comparison purpose. Eventually, the performed experiments findings have corroborated that our remarkable approach, the extended SSFT, has provided prominence performance when compared to its suggested counterparts, in the presence and absence of fiber losses. In specific, when the losses were utterly neglected, our approach has achieved the least sum of squares error, whereas when the losses were considered, this superb approach has demonstrated compatible results with the other proposed approaches, along with rendering the least processing time, which places it in the first rank as the fastest scheme. Moreover, it was the only technique to survive when handling higher values of the GVD parameter. Therefore, the extended SSFT is deemed to be an accurate, straightforward, and fast approach for manipulating this significant problem.

\section{Data Availability}

The data used to support the findings of this study are available upon request from the corresponding author.

\section{Disclosure}

This research was accomplished as a part of the authors' employment at their respected institutions. 


\section{Conflicts of Interest}

The authors declare that they have no potential conflict of interest.

\section{References}

[1] L. F. Mollenauer and J. P. Gordon, Solitons in Optical Fibers: Fundamentals and Applications, Elsevier, 2006.

[2] C. Mahnke, A. Hause, and F. Mitschke, "On the creation of solitons in amplifying optical fibers," International Journal of Optics, vol. 2018, Article ID 9452540, 11 pages, 2018.

[3] S. Y. Al-Dabagh and M. S. Abdalla, "Numerical solutions aspect of nonlinear Schrödinger equation in monomode optical fiber," in 2006 2nd International Conference on Information \& Communication Technologies, vol. 2, pp. 2099-2103, Damascus, Syria, April 2006.

[4] H. Apithy, Y. Bouslimani, and H. Hamam, "Split-step algorithm-based propagation modelling of dark soliton-like pulses," International Journal of Modelling and Simulation, vol. 27, no. 1, pp. 68-73, 2007.

[5] P. Chou, "Fiber optics part 2: single-mode fiber vs. multimode-fiber, Cisco blogs," 2018, https://blogs.cisco.com/sp/ fiberopticspt2singlemultifiber.

[6] D. Felice, "A study of a nonlinear Schrödinger equation for optical fibers,” 2016, https://arxiv.org/abs/1612.00358.

[7] F. Copie, S. Randoux, and P. Suret, "The physics of the onedimensional nonlinear Schrodinger equation in fiber optics: rogue waves, modulation instability and self-focusing phenomena," Reviews in Physics, vol. 5, article 100037, 2020.

[8] Y. Y. Choy, W. N. Tan, K. G. Tay, and C. T. Ong, "Crank-Nicolson implicit method for the nonlinear Schrödinger equation with variable coefficient," AIP Conference Proceedings, vol. 1605, no. 1, pp. 76-82, 2014.

[9] E. Kersale, "Analytic solutions of partial differential equationsMATH3414," School of Mathematics, University of Leeds, 2003.

[10] G. M. Muslu and H. A. Erbay, "A split-step Fourier method for the complex modified Korteweg-de Vries equation," Computers \& Mathematics with Applications, vol. 45, no. 1-3, pp. 503-514, 2003.

[11] D. O. Liceaga, Pseudospectral solutions of reaction-diffusion equations that model excitable media: convergence of solutions and applications, Doctoral dissertation, University of British Columbia, 2007.

[12] C. Harley, "Hopscotch method: The numerical solution of the Frank-Kamenetskii partial differential equation," Applied Mathematics and Computation, vol. 217, no. 8, pp. 40654075, 2010.

[13] H. N. Hassan and H. K. Saleh, "Fourier spectral methods for solving some nonlinear partial differential equations," International Journal of Open Problems in Computer Science and Mathematics, vol. 6, no. 2, pp. 144-179, 2013.

[14] X. Kang, K. Cheng, and C. Guo, “A second-order Fourier pseudospectral method for the generalized regularized long wave equation," Advances in Difference Equations, vol. 2015, no. 1, Article ID 339, 2015.

[15] M. Saleh and E. Kovács, "New explicit asymmetric hopscotch methods for the heat conduction equation," Proceedings of the 1st Online Conference on Algorithms, MDPI, Basel, Switzerland, 2021.
[16] N. G. Farag, A. H. Eltanboly, M. S. El-Azab, and S. Obayya, "Pseudo-spectral approach for extracting optical solitons of the complex Ginzburg Landau equation with six nonlinearity forms," Optik, vol. 254, p. 168662, 2022.

[17] M. Aleshams, A. Zarifkar, and M. H. Sheikhi, "Split-step Fourier transform method in modeling of pulse propagation in dispersive nonlinear optical fibers," in Proceedings of CAOL 2005. Second International Conference on Advanced Optoelectronics and Lasers, 2005, vol. 2, pp. 124-126, Yalta, Ukraine, September 2005.

[18] E. Forestieri and M. Secondini, "Solving the nonlinear Schrödinger equation," in Optical Communication Theory and Techniques, pp. 3-11, Springer, Boston, MA, 2005.

[19] A. V. Ramprasad and M. Meenakshi, "A study on the propagation characteristics of pulses in optical fiber communication systems," in 2006 IFIP International Conference on Wireless and Optical Communications Networks, p. 5, Bangalore, India, April 2006.

[20] S. V. Siddamal, R. M. Banakar, and B. C. Jinaga, "Split step method in the analysis and modeling of optical fiber communication system," in International Conference on Advances in Computing, Communication and Control, pp. 254-261, Springer, Berlin, Heidelberg, 2011.

[21] R. Deiterding, R. Glowinski, H. Oliver, and S. Poole, “A reliable split-step Fourier method for the propagation equation of ultra-fast pulses in single-mode optical fibers," Journal of Lightwave Technology, vol. 31, no. 12, pp. 2008-2017, 2013.

[22] J. Shao, X. Liang, and S. Kumar, "Comparison of split-step Fourier schemes for simulating fiber optic communication systems," IEEE Photonics Journal, vol. 6, no. 4, pp. 1-15, 2014.

[23] R. Deiterding and S. W. Poole, "Robust split-step Fourier methods for simulating the propagation of ultra-short pulses in single-and two-mode optical communication fibers," in Splitting Methods in Communication, Imaging, Science, and Engineering, pp. 603-625, Springer, Cham, 2016.

[24] L. Ladányi, L. Scholtz, and J. Müllerová, "Numerical simulations of dispersion effects in chirped Gaussian and soliton pulses," Optical and Quantum Electronics, vol. 49, no. 3, p. 105, 2017.

[25] H. E. Ibarra-Villalon, O. Pottiez, A. Gomez-Vieyra, J. P. Lauterio-Cruz, and Y. E. Bracamontes-Rodriguez, "Numerical approaches for solving the nonlinear Schrödinger equation in the nonlinear fiber optics formalism," Journal of Optics, vol. 22, no. 4, article 043501, 2020.

[26] A. Semenova, S. A. Dyachenko, A. O. Korotkevich, and P. M. Lushnikov, "Comparison of split-step and Hamiltonian integration methods for simulation of the nonlinear Schrodinger type equations," Journal of Computational Physics, vol. 427, article 110061, 2021.

[27] T. R. Taha and M. I. Ablowitz, "Analytical and numerical aspects of certain nonlinear evolution equations. II. Numerical, nonlinear Schrodinger equation," Journal of Computational Physics, vol. 55, no. 2, pp. 203-230, 1984.

[28] J. Ohlsson, P. Schlatter, C. Mavriplis, and D. S. Henningson, "The spectral-element and pseudo-spectral methods: a comparative study," in Spectral and High Order Methods for Partial Differential Equations, pp. 459-467, Springer, Berlin, Heidelberg, 2011.

[29] L. N. Trefethen, "Spectral methods in MATLAB," in Society for industrial and applied mathematics, Philadelphia, Pa.: Society for Industrial and Applied Mathematics (SIAM, 3600 Market Street, Floor 6, Philadelphia, PA 19104), 2000. 
[30] C. Dall'Agnol, P. L. Natti, E. R. Cirilo, N. M. L. Romeiro, and É. R. T. Natti, "Soliton propagation in lossy optical fibers," 2021, https://arxiv.org/abs/2110.10069.

[31] C. Mas Arabi, "Nonlinear propagation in optical fibers: from soliton radiations to multimode instabilities," Doctoral dissertation, Lille 1, 2018.

[32] M. Eguchi, K. Hayata, and M. Koshiba, "Analysis of soliton pulse propagation in an optical fiber using the finite- element method," Electronics and Communications in Japan (Part II: Electronics), vol. 73, no. 3, pp. 81-91, 1990.

[33] V. E. Zakharov and S. V. Manakov, "On the complete integrability of a nonlinear Schrödinger equation," Theoretical and Mathematical Physics, vol. 19, no. 3, pp. 551-559, 1974.

[34] J. R. Buchanan and Z. Shao, A First Course in Partial Differential Equations, World Scientific Publishing Company, 2017.

[35] J. C. Washburne, A distributed surface temperature and energy balance model of a semi-arid watershed, Doctoral dissertation, The University of Arizona, 1994.

[36] S. C. Tsang and K. W. Chow, "The evolution of periodic waves of the coupled nonlinear Schrodinger equations," Mathematics and Computers in Simulation, vol. 66, no. 6, pp. 551-564, 2004.

[37] A. I. Mahdy, "Fourier pseudospectral solution for a 2D nonlinear paraxial envelope equation of laser interactions in plasmas," Journal of Applied Mathematics and Physics, vol. 4, no. 12, pp. 2186-2202, 2016.

[38] J. M. Carcione, "A generalization of the Fourier pseudospectral method," Geophysics, vol. 75, no. 6, pp. A53-A56, 2010.

[39] P. Suarez, An Introduction to the Split Step Fourier Method Using MATLAB, 2016.

[40] N. G. Farag, A. H. Eltanboly, M. S. EL-Azab, and S. S. A. Obayya, "On the analytical and numerical solutions of the one-dimensional nonlinear Schrodinger equation," Mathematical Problems in Engineering, vol. 2021, Article ID 3094011, 15 pages, 2021.

[41] E. Love and W. J. Rider, "On the convergence of finite difference methods for PDE under temporal refinement," Computers \& Mathematics with Applications, vol. 66, no. 1, pp. 33-40, 2013.

[42] J. A. Machado, A. Babaei, and B. P. Moghaddam, "Highly accurate scheme for the Cauchy problem of the generalized Burgers-Huxley equation," Acta Polytechnica Hungarica, vol. 13, no. 6, 2016.

[43] M. Abdelhakem and Y. H. Youssri, "Two spectral Legendre's derivative algorithms for Lane-Emden, Bratu equations, and singular perturbed problems," Applied Numerical Mathematics, vol. 169, pp. 243-255, 2021.

[44] B. P. Moghaddam and Z. S. Mostaghim, "A novel matrix approach to fractional finite difference for solving models based on nonlinear fractional delay differential equations," Ain Shams Engineering Journal, vol. 5, no. 2, pp. 585-594, 2014.

[45] E. M. Abo-Eldahab, R. Adel, H. M. Mobarak, and M. Abdelhakem, "The effects of magnetic field on boundary layer nano-fluid flow over stretching sheet," Applied Mathematics \& Information Sciences, vol. 15, no. 6, pp. 731-741, 2021.

[46] M. Abdelhakem, T. Alaa-Eldeen, D. Baleanu, M. G. Alshehri, and M. El-Kady, "Approximating real-life BVPs via Chebyshev polynomials' first derivative pseudo-Galerkin method," Fractal and Fractional, vol. 5, no. 4, p. 165, 2021.
[47] M. Abdelhakem, M. Biomy, S. A. Kandil, and D. Baleanu, "A numerical method based on Legendre differentiation matrices for higher order ODEs," Information Sciences Letters, vol. 9, no. 3, p. 3, 2020.

[48] D. Steinley, "Validating clusters with the lower bound for sumof-squares error," Psychometrika, vol. 72, no. 1, pp. 93-106, 2007.

[49] C. F. de Oliveira, P. L. Natti, E. R. Cirilo, N. M. L. Romeiro, and É. R. T. Natti, "Numerical stability of solitons waves through splices in quadratic optical media," Acta Scientiarum Technology, vol. 42, no. 1, article e46881, 2020. 